\title{
Development of the bedsore alarm system using microcomputer for wheelchair users
}

\author{
Takashi Komeda, Hiroyuki Koyama, Tateki Uchida, Masao Miyagi, \\ Kikuo Kanaya ${ }^{1)}$, Sadae Kuroiwa ${ }^{2}$, Susumu Otsuka ${ }^{3)}$ and Hiroyasu Funakubo \\ Shibaura Institute of Technology, Saitama, Japan \\ 1)Yokohama Image System Co., Kanagawa, Japan \\ 2)National Murayama Hospital, Tokyo, Japan \\ 3)National Rehabilitation Center for the Disabled, Saitama, Japan
}

Bedsores is one of the most horrible decease for wheelchair users. When those people are continuously sitting in the same posture, pressure,which is the physical factor, is continuously maintained upon the same area of the body, tissues in that area undergo irreversible changes leading to death and bedsores brake out. It is usually said that prevention is the best strategy for bedsores. Normally, wheelchair users learn the bedsores prevention method from the therapist in hospital and pushup is one of the most useful method to release the pressure. However, if they forget to do the push-up, they become dangerous conditions to occur bedsores. Therefore, they must learn the custom to do push-up in hospital.

In this study, we are developing the system to give a alarm when the wheelchair user forget to do the push-up, and they can learn the timing of push-up as the custom in their daily life after using this system one or two weeks.

This system consists of a flexible sensor sheet and a control box. The sensor sheet consists of flexible print circuit comb-shaped electrodes and pressure-sensitive conductive rubber which chances the electric conductivity continuously corresponding to the applied pressure. Each detecting point is placed in a $12 \times 12$ matrix with an $16 \mathrm{~mm}$ pitch. The size of the sensing area is $176 \mathrm{~mm} \times 176 \mathrm{~mm}$. The detecting point has a pair of $16 \mathrm{~mm}$ square comb-shaped electrodes and a pressuresensitive conductive rubber in $7.4 \mathrm{~mm}$ diameter and $0.5 \mathrm{~mm}$ thickness. The control box has a electric circuit based on micro-computer (Z80), a LED display panel and switches. The electric circuit has a A/D converter to get the pressure value, address decoders to scan the sensor matrix, parallel I/O to put on LED and etc.. We use dry batteries which is attached to the control box to supply the electric power. The size of the control box is $200 \mathrm{~mm} \times 200 \mathrm{~mm} \times 70 \mathrm{~mm}$ and the weight is $500 \mathrm{~g}$. Therefore, this system is a portable type and it is easy to use wheelchair users.

This system can measure the distribution of pressure by the sensor sheet when a wheelchair user sits on it, and show the measured result on the LED panel and also add the pressure value of each points to the memory of the electric circuit every 15 seconds. If the high pressure continuously applies to the same position, this system alarm to the wheelchair user by the buzzer. The level of the high pressure, the time interval of the alarm and the time length of push-up can be changed by three switches.

We confirmed the good operation of this system and will try to check the function through the experiment of wheelchair users. 